Research article

\title{
Circulating soluble Fas levels and risk of ovarian cancer
} Arslan Akhmedkhanov*1,2,4, Eva Lundin ${ }^{5}$, Seth Guller ${ }^{1,3}$, Annekatrin Lukanova6,10, Andrea Micheli ${ }^{8}$, Yuehong $\mathrm{Ma}^{1}$, Yelena Afanasyeva², Anne Zeleniuch-Jacquotte ${ }^{2,4}$, Vittorio Krogh ${ }^{8}$, Per Lenner ${ }^{7}$, Paola Muti ${ }^{9}$, Sabina Rinaldi ${ }^{10}$, Rudolf Kaaks ${ }^{10}$, Franco Berrino ${ }^{8}$, Göran Hallmans ${ }^{6}$ and Paolo Toniolo ${ }^{1,2,4}$

\begin{abstract}
Address: ${ }^{1}$ Department of Obstetrics and Gynecology, New York University School of Medicine, New York, NY, USA, ${ }^{2}$ Department of Environmental Medicine, New York University School of Medicine, New York, NY, USA, ${ }^{3}$ Department of Biochemistry, New York University School of Medicine, New York, NY, USA, ${ }^{4}$ NYU Cancer Institute, New York University School of Medicine, New York, NY, USA, ${ }^{5}$ Department of Medical Biosciences/Pathology, University of Umeå, Umeå, Sweden, ${ }^{6}$ Department of Public Health and Clinical Medicine/Nutritional Research, University of Umeå, Umeå, Sweden, ${ }^{7}$ Department of Oncology, University of Umeå, Umeå, Sweden, ${ }^{8}$ Units of Epidemiology, Istituto Nazionale Tumori, Milan, Italy, ${ }^{9}$ Department of Social and Preventive Medicine, State University of New York at Buffalo, Buffalo, NY, USA and ${ }^{10} H o r m o n e s$ and Cancer Group, International Agency for Research on Cancer, Lyon, France

Email: Arslan Akhmedkhanov* - akhmea01@med.nyu.edu; Eva Lundin - eva.lundin@pathol.umu.se; Seth Guller - gulles01@med.nyu.edu; Annekatrin Lukanova - lukanova@iarc.fr; Andrea Micheli - micheli@istitutotumori.mi.it; Yuehong Ma - may01@med.nyu.edu;

Yelena Afanasyeva - afanay01@med.nyu.edu; Anne Zeleniuch-Jacquotte - jacqua02@med.nyu.edu; Vittorio Krogh - krogh@istitutotumori.mi.it; Per Lenner - per.lenner@oc.umu.se; Paola Muti - muti@buffalo.edu; Sabina Rinaldi - rinaldi@iarc.fr; Rudolf Kaaks - kaaks@iarc.fr;

Franco Berrino - berrino@istitutotumori.mi.it; Göran Hallmans - goran.hallmans@nutrires.umu.se; Paolo Toniolo - toniop01@med.nyu.edu

* Corresponding author
\end{abstract}

Published: 22 December 2003

Received: 25 September 2003

BMC Cancer 2003, 3:33

Accepted: 22 December 2003

This article is available from: http://www.biomedcentral.com/I47/-2407/3/33

(C) 2003 Akhmedkhanov et al; licensee BioMed Central Ltd. This is an Open Access article: verbatim copying and redistribution of this article are permitted in all media for any purpose, provided this notice is preserved along with the article's original URL.

\begin{abstract}
Background: Dysregulation of apoptosis, specifically overexpression of soluble Fas (sFas), has been proposed to play a role in the development of ovarian cancer. The main objective of the present study was to evaluate serum sFas as a potential biomarker of ovarian cancer risk.

Methods: The association between serum sFas levels and the risk of ovarian cancer was examined in a case-control study nested within three prospective cohorts in New York (USA), Umeå (Sweden), and Milan (Italy). Case subjects were I 38 women with primary invasive epithelial ovarian cancer diagnosed between 2 months and 13.2 years after the initial blood donation. Control subjects were 263 women who were free of cancer, and matched the case on cohort, menopausal status, age, and enrollment date. Serum sFas levels were determined using a quantitative sandwich enzyme immunoassay.
\end{abstract}

Results: Serum sFas levels were similar in women subsequently diagnosed with ovarian cancer (median, $6.5 \mathrm{ng} / \mathrm{mL}$; range, 4.4 - 10.2) and in controls (median, $6.8 \mathrm{ng} / \mathrm{mL}$; range, 4.5 - 10.I). Statistically significant trends of increasing serum sFas with age were observed among cases $(r=$ $0.39, p<0.0001)$ and controls $(r=0.42, p<0.0001)$. Compared to women in the lowest third, women in the highest third of serum sFas were not at increased risk of ovarian cancer after adjustment for potential confounders (odd ratio (OR), 0.87; 95\% confidence interval $(\mathrm{Cl}), 0.42$ I.82).

Conclusion: The results suggest that serum sFas may not be a suitable marker for identification of women at increased risk of ovarian cancer. 


\section{Background}

Ovarian cancer remains the most deadly gynecologic tumor in the United States [1] and Europe [2]. In 2003, the American Cancer Society estimated that 25,400 women in the United States will be diagnosed with ovarian cancer, and 14,300 will die from the disease [1]. Accumulating experimental evidence suggests that the dysregulation of apoptosis, specifically a failure to eliminate ovarian epithelial inclusion cysts in the stroma, may play a role in the pathogenesis of the disease $[3,4]$. One of the major regulators of programmed cell death or apoptosis is the Fas/FasL system. Fas (Apo-1, CD95) is a member of the tumor necrosis factor receptor (TNF-R) superfamily [5] and can occur as both a cell-surface and a soluble protein (sFas). The interaction between Fas ligand (FasL) and cell-surface Fas induces apoptosis in sensitive cells through the activation of an intracellular Fas-associated death domain, the recruitment of caspase- 8 and the activation of a cascade of proteases, resulting in proteolysis and degradation of chromosomal DNA [5]. Soluble Fas is usually generated by alternative mRNA splicing and lacks a transmembrane domain. By binding to FasL, sFas is thought to inhibit cell-surface Fas-FasL signaling and downregulate Fas-mediated apoptosis [6]. Elevated serum levels of sFas have been detected in ovarian cancer patients $[4,7,8]$. Serum sFas levels have been shown to correlate directly with advanced stage and increased tumor burden [9] and have been proposed as a prognostic factor in patients with gynecologic malignancies [8]. It has been hypothesized that circulating levels of sFas may be also reflective of risk of subsequent development of ovarian cancer. The objective of the present study was to evaluate the association between serum sFas levels and risk of ovarian cancer using samples collected in advance of clinical diagnosis from three collaborating prospective cohorts.

\section{Methods}

\section{Study cohorts}

The prospective cohorts in this study included the New York University Women's Health Study (NYUWHS), the Northern Sweden Health and Disease Study (NSHDS), and the Italian study of Hormones and Diet in the Etiology of Breast Tumors (ORDET). The cohorts have been described in detail previously [10-12]. Overall study coordination was provided through the International Agency for Research on Cancer (IARC).

At the time of recruitment, cohort members were administered questionnaire focusing on demographic (race, ethnicity, occupation), anthropometric (height, weight, body mass index), reproductive history (age at menarche, age at first full-term pregnancy, age at menopause), family history of cancer, medical history, and medication use. The quality of information collected at baseline was compara- ble between the three participating cohorts. Subjects who reported hormone use at baseline were not eligible in the NYUWHS and the ORDET cohorts, and potential case and control subjects from the NSHDS who reported use of exogenous hormones were also considered ineligible for this study. At recruitment, all cohort members were asked to donate venous blood samples, which have been stored at $-80^{\circ} \mathrm{C}$ for subsequent biochemical analyses. Samples chosen for this study were selected among the specimens that never undergo freeze-thaw cycles previously.

\section{Nested case-control study}

Eligible cases were subjects with primary, invasive epithelial ovarian cancer diagnosed at least 2 months after the initial blood donation and identified within each parent cohort by the date of the last complete follow-up. In the NYUWHS, follow-up consists of periodic (every 2-5 years) contacts of subjects by mail and telephone, as well as record linkages with the statewide tumor registries of New York, New Jersey, Connecticut and Florida, and with the U.S. National Death Index. In the NSHDS, ovarian cancer cases were identified through linkages with regional and national cancer registries and the vital status of the study participants was ascertained by record linkage to the regional and national registries for all-cause mortality. The ORDET database was linked to the regional Lombardy Cancer Registry to identify ovarian cancer cases, and to the regional residents' files to verify the vital status of the cohort members. The dates of last complete follow-up were January 1998, December 2000, and January 1997 for the NYUWHS, NSHDS, and ORDET, respectively. A total of 138 ovarian cancer cases met the eligibility criteria and were included in this study from the three parent cohorts (74 cases from the NYUWHS, 47 cases from the NSHDS, and 17 cases from the ORDET study). Additional serum samples collected during annual examinations were available for a subset of the NYUWHS subjects (30 cases, 59 controls).

For each case, two controls per case were randomly selected among appropriate risk sets. The risk set for a given case included cohort subjects who were alive and free of cancer, have not had a bilateral oophorectomy, and matched the case on cohort, age ( \pm 6 months), date of recruitment ( \pm 3 months), self-reported menopausal status at enrollment, and, for premenopausal women, day of the menstrual cycle at blood donation (for NYUWHS and ORDET subjects only). The goodness of matching for menopausal status was confirmed by FSH measurements (FSH levels $\leq 12.5 \mu \mathrm{IU} / \mathrm{mL}$ were considered premenopausal), as previously described [13,14]. A total of 276 eligible matching controls were identified. Eleven potential controls were excluded because of the lack of serum and two controls were excluded because their FSH levels did not match their reported menopausal status. A total of 
138 ovarian cancer cases and 263 matching controls were included in the study.

The Institutional Review Boards of the New York University School of Medicine, the University of Umeå and Instituto Nazionale Tumori in Milan reviewed and approved the present study.

\section{Laboratory analyses}

The samples from the NSHDS and ORDET were shipped on dry ice to the Reproductive Biology Research Laboratory at the Department of Obstetrics and Gynecology, NYU School of Medicine. Levels of sFas were measured at the NYU School of Medicine laboratory using a quantitative sandwich enzyme immunoassay technique according to manufacturer (Quantikine Human sFas Immunoassay, R\&D Systems Inc., MN). Blood serum samples from each case and her matching controls were always analyzed in the same batch by a laboratory technician who was unaware of their disease status. Concentrations of sFas in nanograms per milliliter $(\mathrm{ng} / \mathrm{mL})$ were calculated using a standard curve generated for each set of samples. All samples were analyzed in duplicate and the average of two measurements was used to calculate serum sFas levels. The assay sensitivity defined as the minimum detectable concentration of sFas is $0.02 \mathrm{ng} / \mathrm{mL}$. The intra-assay and interassay coefficients of variation provided by the laboratory were $3.1 \%$ and $4.5 \%$, respectively. Furthermore, this assay demonstrated no significant cross-reactivity or interference with 92 common cytokines and growth factors.

\section{Statistical analyses}

In order to reduce departures from the normal distribution, sFas levels, weight, height, and body mass index (BMI, calculated as weight in kilograms divided by the square of height in meters) were log-transformed. A mixed effects linear regression model was used to compare continuous variables between cases and controls, taking into account the matched design [15]. Categorical characteristics of cases and controls were compared using the conditional logistic regression model. Spearman correlation coefficients were calculated to quantify the strength of associations between serum sFas levels and various subjects' characteristics. A multivariate linear regression model estimated by the least squares was also used to identify factors predictive of sFas levels.

To compute odds ratios for ovarian cancer, sFas measurements were categorized into tertiles, using the frequency distribution of cases and controls combined. The conditional logistic regression model was used to take into account the matching. Odds ratios were computed relative to the lowest tertile. The effect of potential confounding variables (parity, use of oral contraceptives, and BMI) was assessed by including these variables in the logistic regression model containing sFas one at a time, as well as by including them simultaneously. When assessing the effect of BMI, height was also included in the model because it has been shown that, if obesity is a risk factor, including only BMI in a model forces an inverse-quadratic relation of height to risk given weight [16]. Likelihood ratio tests were used to assess statistical significance and pvalues for trends. Reported trend test $\mathrm{p}$-values correspond to sFas treated as an ordered categorical variable. The statistical analyses were performed using Statistical Analysis Software, Version 8.0 (SAS Institute Inc., Cary, NC).

\section{Results}

Common epithelial-type ovarian tumors were observed, such as serous $(\mathrm{n}=59)$, mucinous $(\mathrm{n}=14)$, endometrioid $(\mathrm{n}=14)$, clear cell $(\mathrm{n}=7)$, mixed $(\mathrm{n}=1)$, and not-otherwise-specified adenocarcinoma $(n=25)$. Histology was unknown for eighteen cases. For all cases combined, median period from the initial visit to the diagnosis of ovarian cancer was 5.1 years (range, 2 months - 13.2 years).

Selected descriptive characteristics of study subjects stratified by participating cohort are presented in Table 1 (see Additional file 1). The median age at diagnosis of ovarian cancer was 61 years in the NYUWHS, 59 years in the NSHDS study, and 54 years in the ORDET study. Because of the matching, ovarian cancer cases and controls had similar proportions of premenopausal and postmenopausal women and similar age at enrollment. There were no significant differences in the ages at menarche or at first full-term pregnancy between the cases and controls. Ovarian cancer cases were more likely to report nulliparity, later age at menopause (all cohorts), a positive first-degree history of breast cancer (the NYUWHS and the ORDET cohorts) and less likely to report use of oral contraceptives (the NSHDS and the ORDET cohorts) as presented in Table 1 (see Additional file 1). Among all subjects, significant case-control differences were observed in parity ( $p=$ $0.02)$ and age at menopause $(p=0.03)$. In agreement with previous report based on the same cohorts [14], in the combined studies analysis ovarian cancer cases had lower BMI (mean, $25.0 \mathrm{~kg} / \mathrm{m}^{2}$ ), as compared to control subjects (mean, $\left.26.0 \mathrm{~kg} / \mathrm{m}^{2}\right)(p<0.04)$.

Table 1 reports the means, medians, standard deviations and ranges of serum sFas levels in ovarian cancer cases and controls. No statistically significant differences in sFas levels were observed between cases and controls in the total study population, within the participating cohorts, and after stratification by menopausal status. Serum sFas levels were slightly higher in the NSHDS subjects, as compared to the NYUWHS and ORDET subjects but the differences between cohorts were not statistically significant (Table $1)$. 
Table 2: Serum sFas levels in ovarian cancer cases and controls from the three participating cohorts

\begin{tabular}{|c|c|c|c|}
\hline \multirow[t]{2}{*}{ Cohort } & \multicolumn{2}{|c|}{ Serum sFas, ng/mL } & \multirow[b]{2}{*}{$P$-value* } \\
\hline & Cases & Controls & \\
\hline \multicolumn{4}{|l|}{ NYUWHS } \\
\hline$n$ & 74 & 145 & 0.37 \\
\hline Mean $(S D)$ & $6.8(2.2)$ & $6.7(2.1)$ & \\
\hline Median (I0th-90th percentiles) & $6.5(4.3-9.8)$ & $6.5(4.2-9.5)$ & \\
\hline \multicolumn{4}{|l|}{ NSHDS } \\
\hline$n$ & 47 & 86 & 0.20 \\
\hline Mean (SD) & $8.1(7.4)$ & $8.0(2.2)$ & \\
\hline Median (I0th-90th percentiles) & $6.6(4.8-10.9)$ & $7.6(5.5-|| .2)$ & \\
\hline \multicolumn{4}{|l|}{ ORDET } \\
\hline$n$ & 17 & 32 & 0.07 \\
\hline Mean $(S D)$ & $6.2(1.3)$ & $6.7(1.3)$ & \\
\hline Median (I0th-90th percentiles) & $6.6(3.9-7.6)$ & $6.4(5.2-8.1)$ & \\
\hline \multicolumn{4}{|l|}{ Total } \\
\hline$n$ & 138 & 263 & 0.51 \\
\hline Mean (SD) & $7.2(4.6)$ & $7.1(2.2)$ & \\
\hline Median (10th-90th percentiles) & $6.5(4.4-10.2)$ & $6.8(4.5-10.1)$ & \\
\hline
\end{tabular}

* Mixed effect linear regression model (after natural log-transformation). SD, standard deviation.

Table 3: Odds ratios of ovarian cancer for tertiles of serum sFas levels

\begin{tabular}{|c|c|c|c|c|}
\hline \multirow[t]{2}{*}{ Serum sFas tertiles, $\mathrm{ng} / \mathrm{mL}$} & \multirow[t]{2}{*}{ Cases n (\%) } & \multirow[t]{2}{*}{ Controls $n$ (\%) } & \multicolumn{2}{|c|}{ OR (95\%Cl) } \\
\hline & & & Unadjusted* & Adjusted** \\
\hline$<5.9$ & $51(37 \%)$ & $83(32 \%)$ & 1.00 & 1.00 \\
\hline $5.9-7.6$ & $46(33 \%)$ & $88(33 \%)$ & $0.86(0.50-1.48)$ & $0.93(0.49-1.78)$ \\
\hline$>7.6$ & $41(30 \%)$ & $92(35 \%)$ & $0.69(0.37-1.28)$ & $0.87(0.42-1.82)$ \\
\hline$p$ for trend $* * *$ & & & 0.24 & 0.71 \\
\hline
\end{tabular}

* Except for matching on cohort, age, menopausal status, and date of blood donation. ** Adjusted for parity, use of oral contraceptives, BMI (logtransformed), and height (log-transformed), *** Conditional logistic regression model (sFas treated as ordered categorical variable).

The associations between serum sFas levels expressed as tertiles and risk of ovarian cancer are reported in Table 2. No statistically significant associations were observed in unadjusted analyses, nor in analyses adjusting for potential confounders (parity, use of oral contraceptives, BMI, and height) included in the statistical model either one at a time, or simultaneously. Both unadjusted and adjusted simultaneously for potential confounders, models are presented.

Because it has been suggested that sFas can be produced and shed into circulation by tumor [9], we explored the hypothesis that sFas levels increase closer to diagnosis with increasing ovarian tumor load and could serve as a marker of yet undiagnosed disease. First, we assessed serum sFas levels stratified by lagtime to the index date (ovarian cancer diagnosis). There were no significant dif- ferences in sFas levels stratified by lagtime to the index date among ovarian cancer cases and their matching controls (Figure 1). We also observed no statistically significant associations between prediagnostic serum sFas and tumor stage $\left(P_{\text {trend }}=0.54\right)$ or grade $\left(P_{\text {trend }}=0.76\right)$.

In addition, we examined the changes over time in sFas levels among subjects for whom repeated blood donations were available ( 30 cases and 59 matched controls from the NYUWHS cohort). For cases, median time between the first and second visits was 376 days, median sFas difference between the first and repeated measurements was $-0.19 \mathrm{ng} / \mathrm{mL}$ and median rate of change was $4.1 \times 10^{-4} \mathrm{ng} / \mathrm{mL} /$ day. For controls, median time between two visits was 378 days, median sFas difference between the first and repeated measurements was $0.10 \mathrm{ng} / \mathrm{mL}$ and median rate of change was $2.8 \times 10^{-4} \mathrm{ng} / \mathrm{mL} /$ day. The 


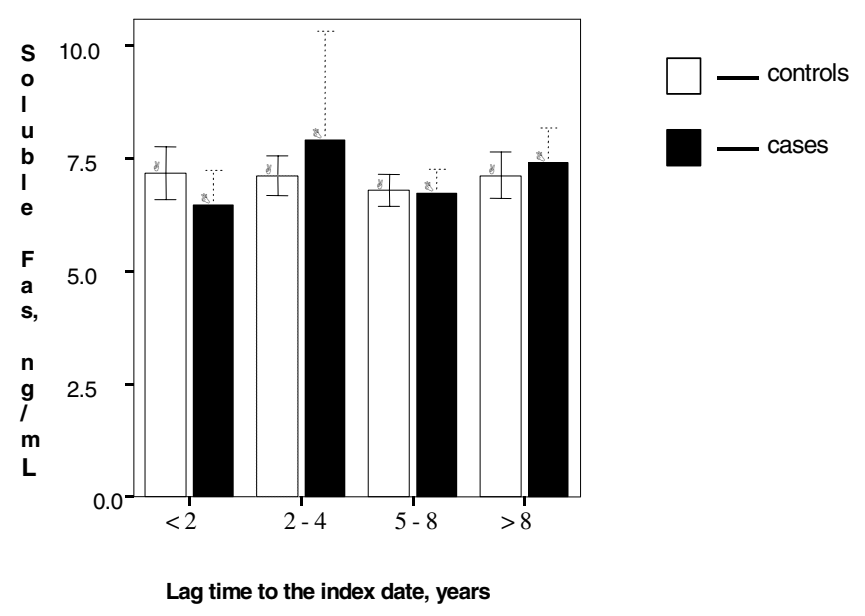

Figure I

Levels of sFas by lagtime from the blood donation to the index date (ovarian cancer diagnosis). Bars show means and $95 \%$ confidence intervals. There were no significant differences in sFas levels stratified by lagtime to the index date among ovarian cancer cases $(p=0.38)$ and matching controls $(p=0.80)$.

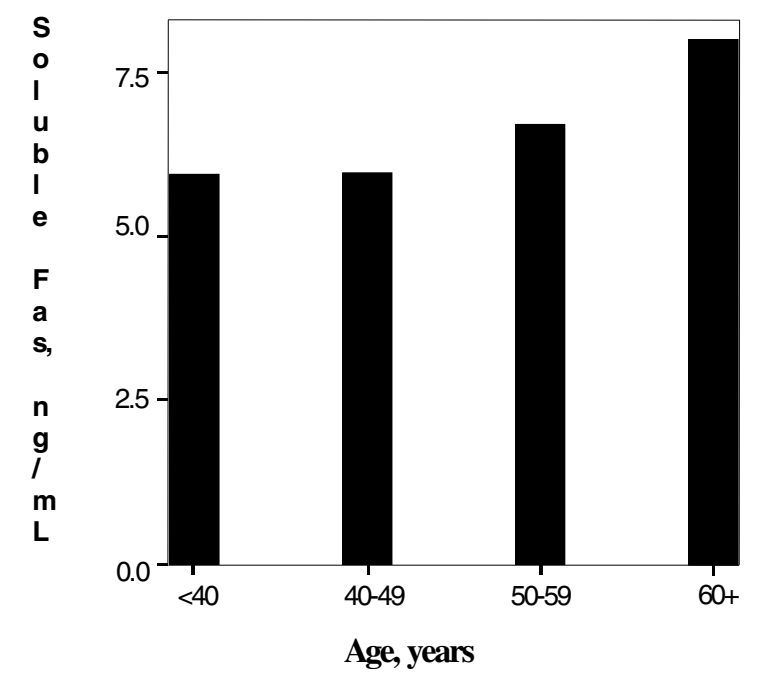

Figure 2

Correlation between age and sFas levels in the control group. There was a significant correlation (Spearman $r=$ $0.42, p<0.000 \mathrm{I}$ ) between age and sFas (median levels presented).

difference in rate of change between ovarian cancer cases and matched controls with repeated blood donations was not statistically significant $(p=0.81)$. Spearman correlation coefficient between first and second sFas measurements was $0.72(p<0.0001)$.

Little is known about factors that determine physiological sFas levels. To identify the factors that may be related to serum sFas, we examined correlations of sFas levels with known characteristics of control subjects. There was a direct and statistically significant correlation between serum sFas and age (Spearman $r=0.42, p<0.0001$ ) (Figure 2). Similar positive correlation between serum sFas and age was observed among cases (Spearman $r=0.39$, $p$ $<0.0001)$. We also analyzed the effect of menopausal status on sFas levels. Results of a linear regression analysis indicated that sFas levels were positively associated with age $(p<0.002)$ but not with menopausal status $(p=0.52)$. After adjustment for age, no significant correlations were observed between serum sFas and selected subjects' characteristics, such as age at menarche, age at first full-term pregnancy, age at menopause, weight, height, and BMI.

\section{Discussion}

The Fas-FasL system is a primary mediator of apoptosis in many major physiologic processes. The proposed roles of Fas-mediated apoptosis include the elimination of activated T-cells following an immune response, the killing of tumor and virally infected cells by cytotoxic T-cells and natural killer cells, and maintenance of immune-privileged sites such as the eye, testis, and maternal-fetal interface.

Dysregulation of Fas-mediated apoptosis is thought to play a role in the development of various tumors, including ovarian cancer $[3,4]$. It has been proposed that cancer cells expressing FasL have an advantage to evade human immune surveillance by inducing apoptosis in infiltrating lymphocytes expressing Fas. It is known that some tumor cells express both Fas and FasL yet are resistant to Fasmediated apoptosis. This phenomenon has been attributed to receptor dysfunction, or intracellular inhibition by FLICE inhibitory protein (FLIP) that blocks Fas-mediated tumor cell death, enabling the tumor to avoid immune clearance [17].

Soluble form of Fas receptor, which is lacking the transmembrane domain of the cell-surface Fas, is thought to compete for FasL with Fas receptor expressed on tumor cells and, therefore, alter or prevent apoptotic signal transduction [6]. Previously, several hospital-based case-control studies have shown that circulating sFas levels were significantly elevated in ovarian cancer patients as compared to controls $[4,7,8]$ and it has been proposed that serum sFas levels or FasL expression may serve as a prognostic factor in patients with ovarian cancer and other gynecological malignancies $[8,18]$. 
The objective of the current study was to evaluate serum sFas as a predictive marker of ovarian cancer risk. In this case-control study nested within prospective cohorts, we observed no association between serum sFas levels and subsequent risk of subsequent ovarian cancer, before and after adjustment for potential confounders.

Several factors should be taken into consideration in the interpretation of these results. The levels of serum sFas observed in our study were markedly higher than in earlier studies $[4,7,8]$. While two of the previous studies $[7,8]$ used sFas ELISA assays (Medical \& Biological Laboratories Co., Nagoya, Japan), the present study used a quantitative sandwich enzyme immunoassay technique (Quantikine Human sFas Immunoassay, R\&D Systems Inc., MN), a very sensitive and specific assay. Therefore, the observed differences in serum sFas levels between the current and previous studies could reflect variations in laboratory assay methods.

The discrepancy in results between this case-control study nested within prospective cohorts and previous retrospective hospital-based studies may also be related to the origins of sFas. Although the precise cellular source of serum sFas remains unclear, increased sFas levels in culture supernatants of breast and colon cancer cells lead to the assumption that sFas is derived from tumor cells [9]. It has been shown that serum sFas are elevated in patients with more advanced tumor stages [7-9] and that serum sFas levels decrease after surgical resection, as compared with levels obtained before surgery [9]. These findings suggest that sFas is produced and shed into circulation by tumor and that serum sFas levels could be reflective of tumor bulk. This may explain the results of previous hospitalbased case-control studies suggesting that sFas can be used as a prognostic marker in patients with advanced ovarian cancer, as well as the lack of association between serum sFas and ovarian cancer observed in this prospective study, for which serum samples were collected up to 13.2 years before diagnosis.

Soluble Fas can also be produced by activated peripheral blood lymphocytes [19] and it has been postulated that dysfunction of apoptotic pathways or production of soluble factors including sFas and soluble FasL may be involved in the pathogenesis of several autoimmune diseases [20] and in cancer [3,4,21]. We observed a strong positive correlation between age and serum sFas suggesting that circulating sFas levels are increasing with age. Because the incidence of cancer and autoimmune disease also increases with age, this observation provides additional indirect evidence for a possible role of apoptosis dysregulation in these conditions. Future studies of sFas should consider taking into account the age-related changes in circulating sFas levels.

\section{Conclusions}

In conclusion, previous studies have demonstrated that serum sFas could be a useful prognostic factor in women who are already diagnosed with ovarian cancer. However, the results of the present study, which observed lack of association between prediagnostic serum sFas and ovarian cancer diagnosed later in life, suggest that serum sFas may not be a suitable susceptibility marker for identification of women at increased risk of the disease.

\section{List of abbreviations}

BMI, body mass index; CI, confidence interval; ELISA, enzyme-linked immunoassay; FasL, Fas ligand; FLIP, FLICE inhibitory protein; FSH, follicle-stimulating hormone; IARC, International Agency for Research on Cancer; ng/mL, nanograms per milliliter; NSHDS, Northern Sweden Health and Disease Study; NYUWHS, New York University Women's Health Study; ORDET, Hormones and Diet in the Etiology of Breast Tumors study; OR, odds ratio; sFas, soluble Fas receptor; TNF-R, tumor necrosis factor receptor.

\section{Competing interests}

None declared.

\section{Authors' contributions}

AA, AL, PT participated in study design and preparation of the manuscript. AA and YA participated in sample preparation from the NYUWHS site. EL and PL participated in sample preparation from the NSHDS site. AM and VK participated in sample preparation from the ORDET site. SR participated in sample preparation and aliquoting at the IARC. SG and YM performed the laboratory analyses. YA and AZ-J participated in the statistical analyses. AA, PM, $\mathrm{RK}, \mathrm{FB}, \mathrm{GH}$ and PT participated in the study coordination, manuscript editing and final manuscript preparation.

\section{Additional material}

\author{
Additional File 1 \\ Characteristics of ovarian cancer cases and controls in the three partici- \\ pating cohorts \\ Click here for file \\ [http://www.biomedcentral.com/content/supplementary/1471- \\ 2407-3-33-S1.doc]
}

\section{Acknowledgements}

Primarily supported by research grant DAMD 17-00-I-0555 from the US Department of Defense. The New York University Women's Health Study (NYUWHS) is supported by Public Health Service grant ROI CA34588, the Northern Sweden Health and Disease Study (NSHDS) is sponsored by the Swedish Cancer Society, and the Italian study of Hormones and Diet in the Etiology of Breast Tumors (ORDET) is sponsored by the Italian Association of Cancer Research (AIRC, rif. 226/0I). We thank the participants of the 
NYUWHS, the NSHDS, and the ORDET, whose invaluable help made this project possible.

\section{References}

I. Society American Cancer: Cancer Facts \& Figures - 2003. American Cancer Society, Inc 2003.

2. Gatta G, Lasota MB, Verdecchia A: Survival of European women with gynaecological tumours, during the period 1978-1989. Eur J Cancer 1998, 34:2218-2225.

3. Ghahremani M, Foghi A, Dorrington JH: Etiology of ovarian cancer: a proposed mechanism. Med Hypotheses 1999, 52:23-26.

4. Baldwin RL, Tran H, Karlan BY: Primary ovarian cancer cultures are resistant to Fas-mediated apoptosis. Gynecol Oncol 1999, 74:265-27I.

5. Nagata S, Golstein P: The Fas death factor. Science 1995, 267: |449-| 456.

6. Owen-Schaub LB, Angelo LS, Radinsky R, Ware CF, Gesner TG, Bartos DP: Soluble Fas/APO-I in tumor cells: a potential regulator of apoptosis? Cancer Lett 1995, 94: I-8.

7. Hefler L, Mayerhofer K, Nardi A, Reinthaller A, Kainz C, Tempfer C: Serum soluble Fas levels in ovarian cancer. Obstet Gynecol 2000, 96:65-69.

8. Konno R, Takano T, Sato S, Yajima A: Serum soluble fas level as a prognostic factor in patients with gynecological malignancies. Clin Cancer Res 2000, 6:3576-3580.

9. Midis GP, Shen Y, Owen-Schaub LB: Elevated soluble Fas (sFas) levels in nonhematopoietic human malignancy. Cancer Res 1996, 56:3870-3874.

10. Toniolo PG, Pasternack BS, Shore RE, Sonnenschein E, Koenig KL, Rosenberg C, Strax P, Strax S: Endogenous hormones and breast cancer: a prospective cohort study. Breast Cancer Res Treat 1991, I 8 Suppl I:S23-S26.

II. Chajes V, Hulten K, Van Kappel AL, Winkvist A, Kaaks R, Hallmans $G$, Lenner P, Riboli E: Fatty-acid composition in serum phospholipids and risk of breast cancer: an incident case-control study in Sweden. Int J Cancer 1999, 83:585-590.

12. Muti P, Pisani P, Crosignani P, Micheli A, Panico S, Secreto G, Berrino F: ORDET--prospective study on hormones, diet and breast cancer: feasibility studies and long-term quality control. Steroids 1988, 52:395-396.

13. Greendale GA, Sowers M: The menopause transition. Endocrinol Metab Clin North Am 1997, 26:26I-277.

14. Lukanova A, Toniolo P, Lundin E, Micheli A, Akhmedkhanov A, Muti P, Zeleniuch-Jacquotte A, Biessy C, Lenner P, Krogh V, Berrino F, Hallmans G, Riboli $E$, Kaaks R: Body mass index in relation to ovarian cancer: a multi-centre nested case-control study. Int J Cancer 2002, 99:603-608.

15. Liang KY, Zeger SL: Longitudinal data-analysis using generalized linear models. Biometrika 1986, 73:13-22.

16. Michels KB, Greenland S, Rosner BA: Does body mass index adequately capture the relation of body composition and body size to health outcomes? Am J Epidemiol 1998, I47:167-I72.

17. French LE, Tschopp J: Defective death receptor signaling as a cause of tumor immune escape. Semin Cancer Biol 2002, I 2:5।-55.

18. Munakata S, Enomoto T, Tsujimoto M, Otsuki Y, Miwa H, Kanno H, Aozasa K: Expressions of Fas ligand and other apoptosisrelated genes and their prognostic significance in epithelial ovarian neoplasms. Br J Cancer 2000, 82: I 446- I 452.

19. Knipping E, Debatin KM, Stricker K, Heilig B, Eder A, Krammer PH: Identification of soluble APO-I in supernatants of human Band $T$-cell lines and increased serum levels in B- and T-cell leukemias. Blood 1995, 85: I562-I569.

20. Mountz JD, Zhou T, Su X, Wu J, Cheng J: The role of programmed cell death as an emerging new concept for the pathogenesis of autoimmune diseases. Clin Immunol Immunopathol 1996, 80:S2-14.

21. O'Connell J, Bennett MW, O'Sullivan GC, Collins JK, Shanahan F: The Fas counterattack: cancer as a site of immune privilege. Immunol Today 1999, 20:46-52.

\section{Pre-publication history}

The pre-publication history for this paper can be accessed here: http://www.biomedcentral.com/1471-2407/3/33/prepub
Publish with Biomed Central and every scientist can read your work free of charge

"BioMed Central will be the most significant development for disseminating the results of biomedical research in our lifetime. "

Sir Paul Nurse, Cancer Research UK

Your research papers will be:

- available free of charge to the entire biomedical community

- peer reviewed and published immediately upon acceptance

- cited in PubMed and archived on PubMed Central

- yours - you keep the copyright

Submit your manuscript here:

http://www.biomedcentral.com/info/publishing_adv.asp
BioMedcentral 